\title{
Adaptive Monitoring and Control Architectures for Power Distribution Grids over Heterogeneous ICT Networks
}

\author{
Rasmus L. Olsen ${ }^{1}$, Christian Hägerling ${ }^{3}$, Fabian M. Kurtz ${ }^{3}$, \\ Florin Iov $^{2}$ and Christian Wietfeld ${ }^{3}$
}

\author{
${ }^{1}$ Department of Electronic Systems, Networking and Security, \\ Aalborg University, Denmark \\ ${ }^{2}$ Department of Energy Technology, Aalborg University, Denmark \\ ${ }^{3}$ Communication Networks Institute, TU Dortmund University, \\ Germany
}

\author{
Received 28 May 2014; Accepted 28 June 2014 \\ Publication 31 August 2014
}

\begin{abstract}
The expected growth in distributed generation will significantly affect the operation and control of today's distribution grids. Being confronted with short time power variations of distributed generations, the assurance of a reliable service (grid stability, avoidance of energy losses) and the quality of the power may become costly. In this light, Smart Grids may provide an answer towards a more active and efficient electrical network. The EU project SmartC2Net aims to enable smart grid operations over imperfect, heterogeneous general purpose networks which poses a significant challenge to the reliability due to the stochastic behaviour found in such networks. Therefore, key concepts are presented in this paper targeting the support of proper smart grid control in these network environments. An overview on the required Information and Communication Technology (ICT) architecture and its functionality is provided and a description of one of several use cases, the External Generation Site is detailed along its evaluation approach.
\end{abstract}

Journal of Communication, Navigation, Sensing and Services, Vol. 1, 151-180.

doi: 10.13052/jconasense2246-2120.123

(C) 2014 River Publishers. All rights reserved. 